\title{
ANALYSIS OF THE INHIBITORY POTENTIAL OF STREPTOCOCCUS SALIVARIUS ISOLATED FROM ADULT SALIVA AND THE TONGUE DORSUM FOR THE GROWTH OF CANDIDA ALBICANS
}

\author{
NINDY FAIRISKA, BOY M BACHTIAR, FERRY P GULTOM*
}

Department of Oral Biology Dentistry, Faculty of Dentistry, Universitas Indonesia, Jakarta, Indonesia. Email: gultom_ferry@yahoo.com Received: 21st April 2017, Revised and Accepted: 18th August 2017

\section{ABSTRACT}

Objectives: The objective of this study is to analyze the effectiveness of Streptococcus salivarius and its protein for inhibiting the growth of Candida albicans.

Methods: The analysis was conducted using polymerase chain reaction, sodium dodecyl sulfate polyacrylamide gel electrophoresis, a Bradford test, deferred antagonism test, and well-diffusion agar.

Result: $S$. salivarius, isolated from saliva and the tongue dorsum, and its protein do not inhibit the growth of C. albicans. The morphology of $C$. albicans did not change after being exposed to protein produced by $S$. salivarius.

Conclusions: $S$. salivarius and its protein do not inhibit the growth of $C$. albicans. However, the bacterium has the capacity to maintain fungus morphology in the form of blastospora.

Keywords: Bacteriocins, Candida albicans, Probiotic, Protein, Streptococcus salivarius.

(C) 2017 The Authors. Published by Innovare Academic Sciences PvtLtd. This is an open accessarticle under the CC BY license (http://creativecommons. org/licenses/by/4. 0/) DOI: http://dx.doi.org/10.22159/ijap.2017.v9s1.05

\section{INTRODUCTION}

Oral health problems are common in Indonesia and have become more complex, resulting in the need for a variety of treatments. Such treatments can be conventional, such as using antibiotics, but overuse of antibiotic can lead to antibiotic resistance, which is a global health concern, both clinically and publically [1]. Therefore, probiotic agents have been developed as natural alternatives to oral health treatments. According to the World Health Organization, a probiotic is a living microorganism that is useful for maintaining health [2]. Probiotics kill or inhibit pathogenic bacterial growth by producing bacteriocins or other products that act antagonically on pathogenic bacteria. One such probiotic is Streptococcus salivarius [3]. S. salivarius originates in the womb, is obtained from an infant's mother several hours after birth, and can be identified 2 days after birth [2]. This bacteria is usually isolated from saliva and almost all surfaces of the oral cavity, especially the tongue and mucus. Known strains include K12, M18, K58, and TOVE-R. K12 S. salivarius can bond with Candida albicans hyphae to prevent the bacteria from adhering to plastic substrate [4]. This strain has been used in New Zealand as an oral isolate probiotic to obstruct in vitro growth of C. albicans and has been shown to have the protective ability in an oral candidiasis model [5].

Oral candidiasis is an opportunistic and common fungal infection caused by $C$. albicans [6]. C. albicans is a component of normal microflora in the oral cavities of $30-50 \%$ of humans and is a dimorphic fungi, meaning it has two morphologies: A yeast type and a mold type (hyphae) [5]. Oral candidiasis caused by $C$. albicans can be treated by applying antifungal agents, such as nystatin and fluconazole [6]; however, there is a chance that probiotic agents can also decrease the growth of $C$. albicans. Protein secreted by $S$. salivarius is a possible natural alternative to antibiotics. Even though the probiotic effects of $S$. salivarius are known, no research has been conducted to identify the protein secreted by $S$. salivarius or inhibitory potential for $C$. albicans growth. Therefore, this research analyzes $S$. salivarius and its protein, isolated from the saliva and tongue dorsum of healthy adult subjects, to determine the inhibitory potential for C. albicans growth.

\section{METHODS}

Samples were swabbed from the dorsum of the tongue and saliva of 10 healthy adult subjects between 19 and 21 years of age [7]. A centrifuge tube (15 ml, $50 \mathrm{ml}$ ), cytobrush, $1000 \mathrm{ml}$ tipped pipet, $200 \mathrm{ml}$ tipped pipet, micropipette, Eppendorf tube, sterilized reaction tubes, 96well microplate, incubator, anaerobic jar, vortex, mini centrifuge, autoclave, centrifuge, orbital shaker, enzyme-linked immunosorbent assay plater reader, analytic scale, water bath, round end ose berujung bulat, light lamp, Petri dishes, thermal cycler T100, electrophoresis, Gel Doc, Thermoblock, sodium dodecyl sulfate polyacrylamide gel electrophoresis (SDS-PAGE) chamber, SDS-PAGE tank, and AxioCam ERc5s (Zeiss) microscope were the tools used to conduct this study. Additional materials included a brain-heart infusion (BHI) broth, BHI agar, sabouraud dextrose agar, bakto agar (Biomatic), mitis salivarius agar (MSA), sterilized aquadest, aquabides, cotton, aluminum foil, $70 \%$ alcohol, gas mixture $\left(\mathrm{N}_{2}, \mathrm{CO}_{2}: 10 \% ; \mathrm{H}_{2}: 10 \%\right)$, phosphate buffer saline, primer Sal-AUS (5'-GTAGAAAATATTTACTACATACT) [8], primer Sal-ADS (5'-GTTAAAGTATTCGTAAAACTGATG) [8], nucleus-free water, Dream Taq, Agarose, gel red, Tris-acetate-ethylenediaminetetraacetic acid buffer, $100 \mathrm{bp}$ ladder, $10 \mathrm{mM}$ Tris-HCl pH 7.4 liquid, $0.1 \mathrm{M}$ TrisHCL pH 6.8 liquid, phenyl methyl sulfonyl fluoride, glycerol, Coomassie brilliant blue, albumin protein standard, acrylamide $40 \%$, stacking gel buffer, resolving gel buffer, SDS 10\%, ammonium persulfate, tetra methyl ethylene diamine, marker protein (Peqlab), MiliQ water, Laemmli buffer, $\beta$-mercaptoethanol, SDS running buffer, $C$. albicans ATCC 10231 glycerol stocks, and S. salivarius ATCC 13419 glycerol stocks.

Subjects were chosen using a non-probability, judgement or purposive sampling method, meaning that the researcher chose participants based on subjective assessments [9]. Samples of $10 \mathrm{ml}$ were taken from stimulated saliva using parafilm M and from the dorsum of subjects' tongues using a cytobrush from the circumvallate papillae to the end of the tongue [10]. The samples were cultured in an MSA medium, and selective media for Streptococci were used to colonize S. salivarius strain of Barbour and Philip and Santagati et al. [11,12]. 
Morphology was identified by observing the size and surface consistency (i.e., soft or smooth) of colonies, which were confirmed as S. salivarius using the polymerase chain reaction (PCR) technique [13]. The presence of 118 base-couple DNA bands indicated that colonies were S. salivarius. These colonies were bred in a Mueller-Hinton infusion agar medium and BHI liquid for 18 hrs. The results were centrifuged, and pellets were made from the material separated from the spent medium protein using a cell lysate buffer and centrifugation. These were analyzed using SDS-PAGE (150 V; $80 \mathrm{~mA}$ for 60 minutes) [14]. Proteins with the same molecular mass were counted using a Bradford test [15]. $S$. salivarius inhibitory potential against $C$. albicans was tested using a deferred antagonism test [3], and secreted proteins were tested using the well-diffused agar method. This method used whole-cell and spent medium protein from clinically obtained $S$. salivarius and $S$. salivarius ATCC 13419 [16].

Independent variables were healthy adult saliva and dorsum tongue swabs. Dependent variables were $S$. salivarius inhibitory potential against C. albicans 10231, protein secreted by $S$. salivarius, and C. albicans morphological changes. The controlled variable was $S$. salivarius ATCC 13419 . Data were analyzed by comparing $S$. salivarius colonies identified in isolated saliva and dorsum tongue swabs to colonies that did not contain $S$. salivarius from the same sources. Data were tested using a Fisher's test, and the means of $S$. salivarius inhibitory proteins for $C$. albicans growth in each isolated source were compared.

\section{RESULTS}

Colonies were identified by comparing the morphology of S. salivarius cultured from subject samples to the morphology of $S$. salivarius ATCC

Table 1: $S$. salivarius isolate identifications

\begin{tabular}{lll}
\hline Isolated sources & \multicolumn{2}{l}{ S. salivarius colonies } \\
\cline { 2 - 3 } & $+\mathbf{( \% )}$ & $-(\%)$ \\
\hline Saliva & $6(60)$ & $4(40)$ \\
Tongue dorsum & $6(60)$ & $4(40)$ \\
\hline
\end{tabular}

S. salivarius: Streptococcus salivarius
13419 grown on an MSA medium. From six saliva and tongue dorsum samples, similar morphologies to $S$. salivarius ATCC were obtained (Table 1), and a PCR test was conducted to confirm this morphology. $S$. salivarius interaction with $C$. albicans was tested using the crossstreak method (Fig. 1) and a deferred antagonism test. Results for the inhibition zone diameter produced by $S$. salivarius against $C$. albicans were taken in millimeters (mm). From four subjects, only one subject's results could be interpreted; therefore, only saliva and tongue dorsum samples from this one subject were used.

Table 2 shows that in all isolated $S$. salivarius concentrations and the $S$. salivarius ATCC 13419 control, the inhibition zone mean was 0 $\mathrm{mm}$. Protein secreted by $S$. salivarius had either whole-cell or spent medium protein inhibition potential against $C$. albicans, which was tested using the well-diffused agar method. Table 3 shows that the mean inhibition zone of $S$. salivarius concentrations from isolated sources was $0 \mathrm{~mm}$.

Table 4 shows that the inhibition zone around each well of protein concentrate isolated from either source was $0 \mathrm{~mm}$. C. albicans was taken using the well-diffused agar method and observed microscopically to obtain morphological images. Table 5 shows that $C$. albicans morphologic images without protein exhibited germ tube changes, while $C$. albicans exposed to the protein remained blastospores.

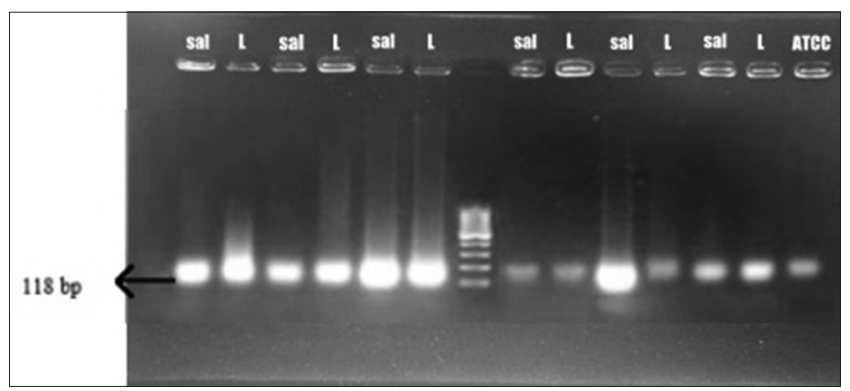

Fig. 1: Polymerase chain reaction results for Streptococcus salivarius isolated from the saliva and tongue dorsum. Sal: Saliva isolated; L: Tongue dorsum isolated; ATCC: $S$. salivarius ATCC 13419

Table 2: The mean for the inhibition zone of each isolate concentration based on a deferred antagonism test

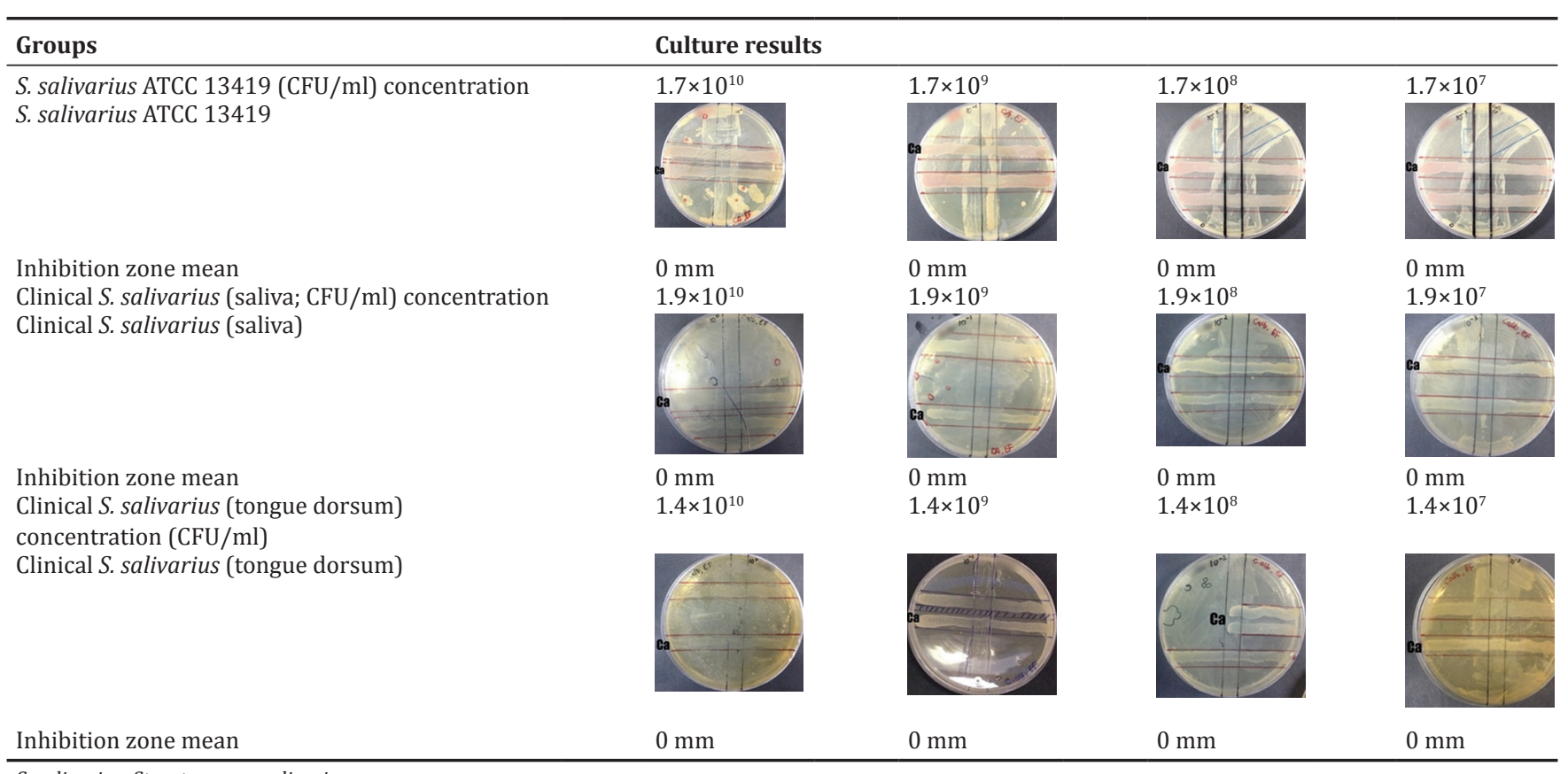

S. salivarius: Streptococcus salivarius 
Table 3: The mean inhibition zone for concentrations of whole-cell isolate using the well-diffused agar method

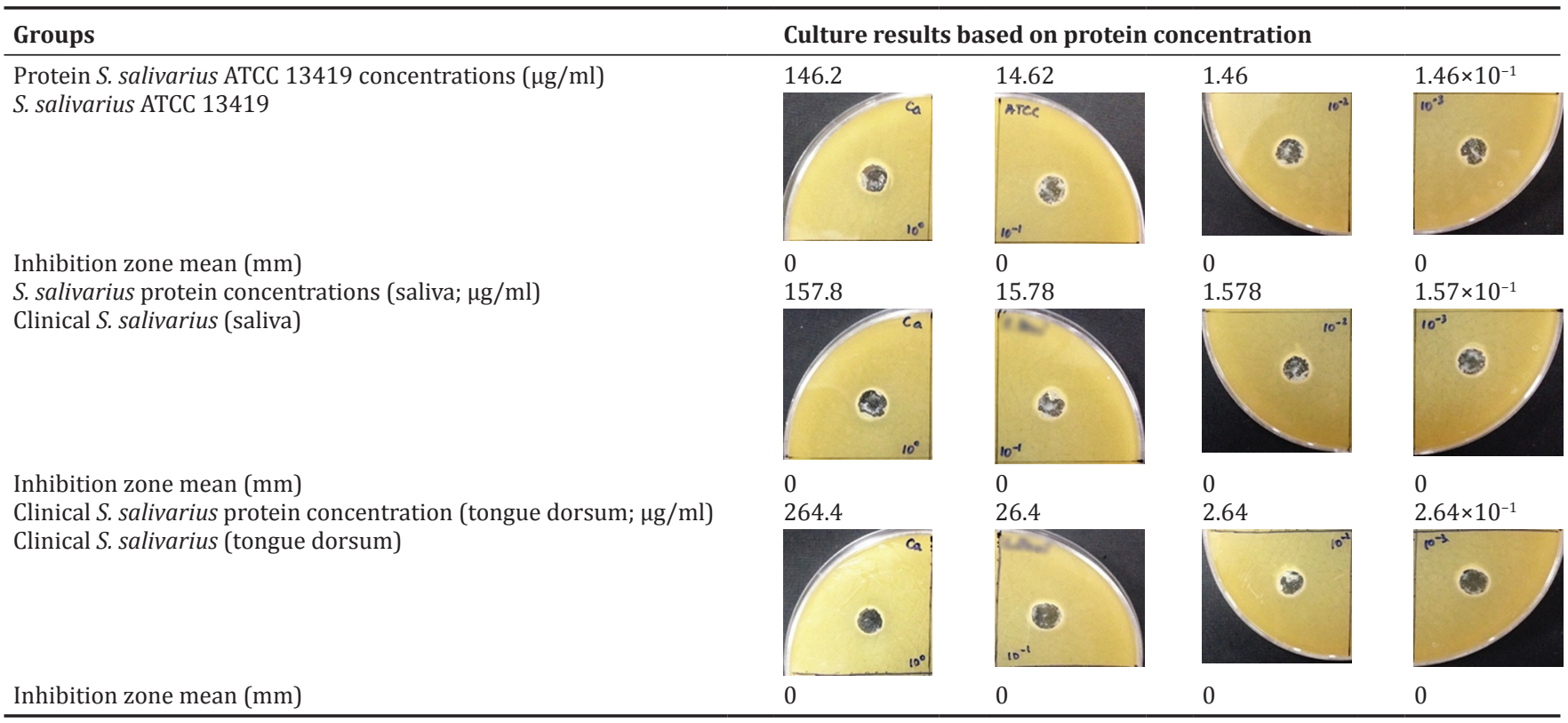

S. salivarius: Streptococcus salivarius

Table 4: The mean inhibition zone for protein concentrations in a spent medium isolate using the well-diffused agar method

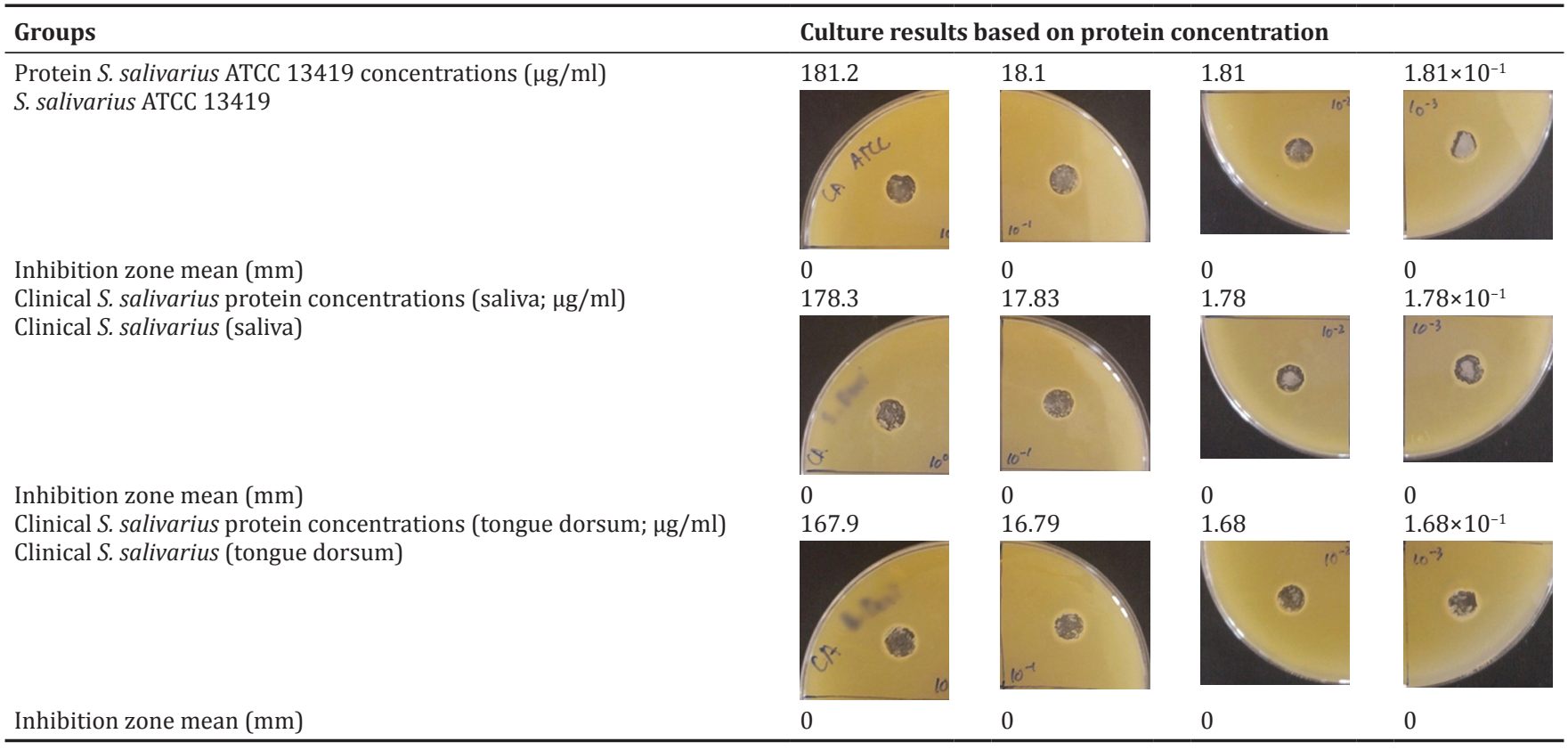

S. salivarius: Streptococcus salivarius

\section{DISCUSSION}

This research identified S. salivarius using cultured samples in an MSA medium using PCR tests. Results showed that from 10 samples, six subjects ( $p>0.05$ ) were $S$. salivarius positive in both saliva and tongue dorsum samples. These results are consistent with the results of Ogawa et al. (2010) [22] and Wescombe et al. who stated that $S$. salivarius is a pioneer bacteria in the oral cavity and has predominant bacterial lifespan $[4,16]$. Although there was no significant statistical difference, low concentrations of $S$. salivarius found in this research were caused by certain factors. The absence of $S$. salivarius in four subjects was due to these subjects' diets. Ogawa et al. (2010) stated that diet can cause a specific microbiota composition due to the intrinsic capacity of each person to use consumed substrate. For example, consuming polysaccharides causes different microbiota compositions. S. salivarius requires energy from sucrose to grow and colonize [16]; thus, another study found that sucrose influenced S. salivarius prevalence in adults. When sucrose is eliminated from the diet, concentrations of S. salivarius in saliva drastically decrease [17].

In addition, Roger et al. [17] stated that enzymes in saliva can influence $S$. salivarius growth in the oral cavity. These enzymes, including lysozyme, lactoperoxidase, and amylase, have antibacterial activities [17] that can reduce $S$. salivarius growth. In this research, subjects were allowed to eat before sampling, which was performed 3 hrs after a meal. Subjects also brushed their teeth beforehand; therefore, diet differences, enzymes contained in saliva, and samplestaking procedures all affected the results obtained in this study. 
Table 5: Microscopic images of C. albicans taken using the well-diffused agar method

\begin{tabular}{lccc}
\hline Groups & Controlled & ATCC 13419 & Saliva \\
\hline Whole-cell protein & 0 & & \\
& 0 & 0 & \\
Spent medium protein & 0 & 0 & \\
& & 0 & 0 \\
\hline
\end{tabular}

C. albicans: Candida albicans

The specific protein secreted by $S$. salivarius colonies was identified using an SDS-PAGE test. Protein used in this test was presented as whole-cell protein (i.e., protein inside cellular membranes) and spent medium (i.e., protein secreted outside the cell). Barbour and Philip (2014) stated that $60-70 \%$ of bacteriocin are peptides that bond with bacterial cell-producer walls, while other inhibitory peptides are secreted extracellular in liquid mediums. Secreted peptides are dominant in antibiotic production because they act as communication molecules that accumulate during the growth process. In some concentrations, lantibiotic production is triggered [11]. According to Wescombe et al. [8], salivaricin A production can be detected in individuals' saliva in varying concentrations because there is a variety of salivary flow rates that dissolve salivaricin A and allow adsorption in normal cell flora [8]. SDS-PAGE results in the current study showed that from six $S$. salivarius samples, only four subjects produced protein with the same profiles, containing one band located inline. The absence of spent medium protein could be due to the communication mechanism not triggering protein production. An S. salivarius inhibition potential analysis was conducted to isolate it from the saliva and tongue dorsum samples from one subject, as well as S. salivarius ATCC 13419 and C. albicans, using the deferred antagonism test method and cross streak test. The results revealed that $C$. albicans growth was not inhibited by $S$. salivarius ATCC 13419 concentrations from either saliva or the tongue dorsum.

According to Morales and Hogan [18], bacteria and fungi have structural shapes, and the surfaces can interact to form communities known as biofilms. The interaction between $C$. albicans and oral Streptococci aggregates on cellular surfaces and forms a mixed-species biofilm [18]. According to Shirtliff et al. [19], the interaction between C. albicans and Streptococci gives synergistic results, in that Streptococci produces lactacyd acid that acts as a carbon source for Candida growth. In turn, $C$. albicans reduces oxygen pressure to a more suitable level for Streptococci, which stimulates bacterial growth due to nutritional metabolic support $[19,20]$.

To analyze S. salivarius microbial activity further, inhibition potential tests were conducted on the protein secreted by $S$. salivarius against C. albicans using the well-diffusion agar method. S. salivarius produces bacteriocin, primarily lantibiotic, in the form of salivaricin [4]. The cell lysate method was used to obtain whole-cell protein, and spent medium protein was obtained by centrifugation. Similar to earlier results, protein inhibition potential tests showed that there was no $C$. albicans growth inhibition from either saliva-isolated or tongue-dorsumisolated protein or ATCC 13419 concentrations of either whole-cell or spent medium proteins. Ishijima et al. [5] found that $S$. salivarius K12 is effective against in vitro-cultured $C$. albicans growth because $S$. salivarius K12 bonded directly to Candida, inhibiting adhesion to a plastic Petri dish. S. salivarius K12 bonded to Candida during germ tube formation, and no Candida hyphae form was found [5]. In the current research, microscopic images were obtained to identify $C$. albicans morphology, which showed that controlled $C$. albicans entered the germ tube stage, while $C$. albicans exposed to either whole-cell or spent medium protein remained in the blastospore stage. This finding was supported by Ishijima et al. [5], who found that $S$. salivarius K12 reduced $C$. albicans' ability to maintain its blastospore shape. Thus, S. salivarius does not kill $C$. albicans but interacts and bonds with $C$. albicans hyphae and cannot inhibit $C$. albicans growth based on a deferred antagonism test, which showed that bacteriocin coded from the K12 strain did not target yeast $[4,5]$. MacDonald showed that $S$. salivarius K12 and M18 strains do not reduce yeast growth but inhibit hyphae formation and adhesion to surfaces of yeast [21]. Spent medium protein from S. salivarius K12 and M18 strains also significantly reduced $C$. albicans adhesion to wells and inhibit transition to hyphae form. This is consistent with results from Köhler et al. [9], in which lactobacilli probiotic influenced the C. albicans metabolism and increased in relation to stress. Similarly, fungi were also challenged by Lactobacilli acid production, which reduced $\mathrm{pH}[9,21]$.

According to research by Köhler et al. [9], spent medium protein from probiotics have lower $\mathrm{pH}$ levels than $C$. albicans in normal growth media; thus, reducing $\mathrm{pH}$ can also reduce $C$. albicans adhesion. Stable spent medium $\mathrm{pH}$ does not reduce adhesion significantly, but decreasing spent medium $\mathrm{pH}$ decreases $C$. albicans adhesion. In addition, low $\mathrm{pH}$ levels support $C$. albicans yeast growth and inhibit hyphae formation $[9,21]$. In this research, protein secreted by $S$. salivarius was determined to have the potential to inhibit $C$. albicans morphological changes by maintaining the blastospore form, presumably due to low pH levels and related stress increases that influence $C$. albicans metabolic activity.

\section{CONCLUSION}

This research showed that $S$. salivarius, obtained from saliva or tongue dorsum samples, does not have a potential inhibitory effect on $C$. albicans growth. Either $S$. salivarius whole-cell or spent medium protein secreted from saliva or the tongue dorsum does have the potential to inhibit $C$. albicans morphological changes from blastospore to hyphae. It is suggested that purifying lantibiotic secreted by $S$. salivarius or using an auto-induction method could be used to obtain specific lantibiotic types. The K12 S. salivarius strain could be positively controlled in the future research, and further analysis of lantibiotic as a probiotic to prevent and treat oral candidiasis is necessary. The therapeutic effects of lantibiotic against $C$. albicans could be determined by conducting a candidiasis oral analysis.

\section{REFERENCES}

1. Levy SB. The 2000 Garrod lecture. Factors impacting on the problem of antibiotic resistance. J Antimicrob Chemother 2002;49(1):25-30.

2. Horz HP, Meinelt A, Houben B, Conrads G. Distribution and persistence of probiotic Streptococcus salivarius K12 in the human oral cavity as determined by real-time quantitative polymerase chain reaction. Oral Microbiol Immunol 2007;22(2):126-30.

3. Fauzi AA, Shafiei Z, Baharin B, Mohd N. Isolation of Lactobacillus from periodontally healthy subjects and its antimicrobial activity against periodontal pathogens. Sains Malays 2013;1:19-24.

4. Wescombe PA, Hale JD, Heng NC, Tagg JR. Developing oral probiotics from Streptococcus salivarius. Future Microbiol 2012;7(12):1355-71. 
5. Ishijima SA, Hayama K, Burton JP, Reid G, Okada M, Matsushita Y, et al. Effect of Streptococcus salivarius K12 on the in vitro growth of Candida albicans and its protective effect in an oral candidiasis model. Appl Environ Microbiol 2012;78(7):2190-9.

6. Samaranayake L. Essential Microbiology for Dentistry. $4^{\text {th }}$ ed. Toronto: Churchill Livingstone Elsevier Ltd.; 2012.

7. Yamaguchi M, Tezuka Y, Takeda K, Shetty V. Disposable collection kit for rapid and reliable collection of saliva. Am J Hum Biol 2015;27(5):720-3.

8. Wescombe PA, Upton M, Dierksen KP, Ragland NL, Sivabalan S, Wirawan RE, et al. Production of the lantibiotic salivaricin a and its variants by oral streptococci and use of a specific induction assay to detect their presence in human saliva. Appl Environ Microbiol 2006;72(2):1459-66.

9. Köhler GA, Assefa S, Reid G. Probiotic interference of Lactobacillus rhamnosus GR-1 and Lactobacillus reuteri RC-14 with the opportunistic fungal pathogen Candida albicans. Infect Dis Obstet Gynecol 2012;2012:14.

10. Salimetrics LL. Saliva collection and handling advice. Methods 2009;44:14

11. Barbour A, Philip K. Variable characteristics of bacteriocin-producing Streptococcus salivarius strains isolated from Malaysian subjects. PLoS One 2014;9(6):e100541.

12. Santagati M, Scillato M, Patanè F, Aiello C, Stefani S. Bacteriocinproducing oral streptococci and inhibition of respiratory pathogens. FEMS Immunol Med Microbiol 2012;65(1):23-31.

13. Bowe WP, Filip JC, DiRienzo JM, Volgina A, Margolis DJ. Inhibition of propionibacterium acnes by bacteriocin-like inhibitory substances (BLIS) produced by Streptococcus salivarius. J Drugs Dermatol
2006;5(9):868-70.

14. Johansson A. Aggregatibacter actinomycetemcomitans leukotoxin: A powerful tool with capacity to cause imbalance in the host inflammatory response. Toxins (Basel) 2011;3(3):242-59.

15. Prasad S, Morris PC, Hansen R, Meaden PG, Austin B. A novel bacteriocin-like substance (BLIS) from a pathogenic strain of Vibrio harveyi. Microbiology 2005;151:3051-8.

16. Yang E, Fan L, Jiang Y, Doucette C, Fillmore S. Antimicrobial activity of bacteriocin-producing lactic acid bacteria isolated from cheeses and yogurts. AMB Express 2012;2(1):48.

17. Roger P, Delettre J, Bouix M, Béal C. Characterization of Streptococcus salivarius growth and maintenance in artificial saliva. J Appl Microbiol 2011;111(3):631-41

18. Morales DK, Hogan DA. Candida albicans interactions with bacteria in the context of human health and disease. PLoS Pathog 2010;6(4):e1000886.

19. Shirtliff ME, Peters BM, Jabra-Rizk MA. Cross-kingdom interactions: Candida albicans and bacteria. FEMS Microbiol Lett 2009;299(1):1-8.

20. Diaz PI, Xie Z, Sobue T, Thompson A, Biyikoglu B, Ricker A, et al. Synergistic interaction between Candida albicans and commensal oral streptococci in a novel in vitro mucosal model. Infect Immun 2012;80(2):620-32.

21. MacDonald KW. The Role of Streptococcus salivarius as a Modulator of Homeostasis in the Oral Cavity. Electronic Thesis and Dissertation Repository. University of Western Ontario; 2015.

22. Ogawa A, Furukawa S, Fujita S, Mitobe J, Kawarai T, Narisawa N, et al. Inhibition of streptococcus mutans biofilm formation by streptococcus salivarius FruA. Appl Environ Microbiol. 2011;77(5):1572-80. 